\title{
Nonreciprocal Transmission and Low-Threshold Bistability in Strongly Modulated Asymmetric Nonlinear WBGs
}

\author{
Masafumi Fujii, Tetsuya Takashima, \\ Department of Electrical, Electronic and System Engineering, University of Toyama, 3190 Gofuku, Toyama, 930-8555 Japan \\ Tel/Fax: +81 76445 6762, E-mail: mfujii@eng.u-toyama.ac.jp \\ Ayan Maitra, Juerg Leuthold, Wolfgang Freude \\ Institute of High-Frequency and Quantum Electronics (IHQ), University of Karlsruhe, Germany \\ Christopher Poulton \\ Max-Planck Research Group for Optics, Information and Photonics, University of Erlangen-Nuremberg, Germany
}

\begin{abstract}
Nonreciprocal optical bistability is numerically investigated in InGaAsP/InP nonlinear waveguide Bragg gratings having a strong and asymmetric sidewall modulation. Minimum switching power as low as $77 \mathrm{~mW}$ is predicted by choosing optimal switching conditions.
\end{abstract}

\section{Introduction}

For ultra-fast optical communication, all-optical switches and logic gates are important [1], which can be realized by optical bistability in nonlinear media [2], [3], [4]. In this paper we investigate numerically an optical Schmitt trigger operation in longitudinally asymmetric waveguide Bragg gratings (WBGs). These WBGs have also a property of non-reciprocal transmission, where the switching threshold in one direction is lower than that in the other direction. The analyzed WBG can be fabricated with an optical planar circuit technology using InGaAsP/InP [5]. The sidewall modulation of this structure is large, and thereby the coupled mode equations can not be directly applied due to the strong coupling between the forward and the backward waves [6]. Analysis has been therefore performed fully numerically with the nonlinear finite-difference time-domain (FDTD) method that inherently deals with the necessary coupling and guiding effects of the WBGs.

\section{Nonlinear WBG Configuration}

The asymmetric WBG has been modeled such that the stopband of the grating has a nearly linear profile along the waveguide. The grating is realized by a sidewall periodic perturbation of a dielectric waveguide whose top view and cross section are shown in Fig.1 (a) and (b), respectively. The sidewall modulation is expressed by a function of distance $z$ as

$$
x(z)= \pm\left[\frac{W}{2}+\frac{W_{g}}{2} g\left(z / L_{0}\right) \sin \left(\frac{2 \pi z}{\Lambda}\right)\right],
$$

where $\Lambda$ is the period of the grating chosen to be $\Lambda=0.241 \mu \mathrm{m}$ for this paper, and $L_{0}$ is the total length of the grating. The averaged width of the waveguide was chosen to be $W=0.4 \mu \mathrm{m}$ such that it supports only the dominant propagation mode at an operating frequency. The nearly linear variation of the band edge frequency is described by a 3rd-order polynomial in terms of the normalized distance $z^{\prime}=z / L_{0}$ as $g\left(z^{\prime}\right)=a z^{\prime 3}+b z^{\prime 2}+c z^{\prime}+d$, with $a=-0.4856, b=-0.0009, c=0$, and $d=1$. Note that this results in a chirp of the stopband center frequency along the grating. For $W_{g}=0.1 \mu \mathrm{m}$ and $W_{g}=0.2 \mu \mathrm{m}$, the transmission spectra for the whole asymmetric WBGs is shown in Fig.2. The waveguide has a three-dimensional (3D) pedestal waveguide structure [5], which consists of an InP cladding and an InGaAsP core, and it is approximated by a slab waveguide for analysis. The third-order nonlinear susceptibility for the InGaAsP core is $\chi_{0}^{(3)}=3.8 \times 10^{-18} \mathrm{~m}^{2} / \mathrm{V}^{2}$.

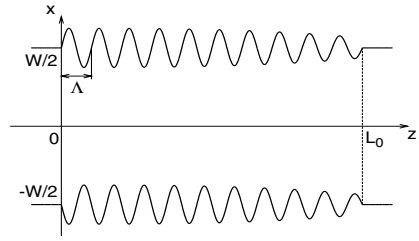

(a)

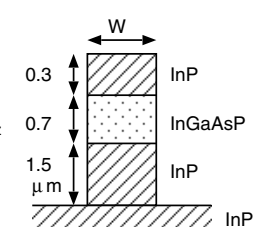

(b)

Fig.1 Schematic configuration of the asymmetric waveguide Bragg grating; (a) the top view, and (b) the cross section of the waveguide.

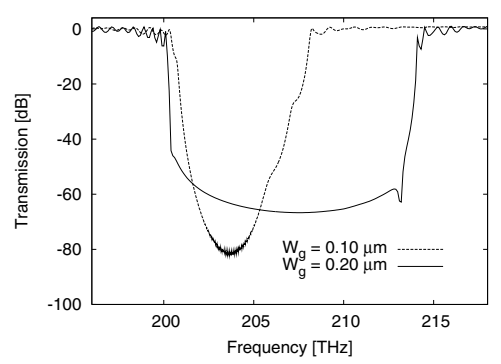

Fig.2 Comparison of the calculated transmission spectra for 200- $\Lambda$-long asymmetric gratings with the maximum modulation $W_{g}=0.1 \mu \mathrm{m}$ and $W_{g}=0.20 \mu \mathrm{m}$. 


\section{FDTD Analysis of Nonlinear WBGs}

We apply Yee's FDTD algorithm to Maxwell's equations [7] including Kerr nonlinearity and typical chromatic dispersion properties through the auxiliary differential equation (ADE) technique [8]. The relation between the length of the waveguide grating and the switching threshold for the RTL configuration is plotted in Fig.3. The switching threshold no longer exhibits a linear dependence on the grating length. This is considered to be due to the strong modulation of the grating.

Next we increased the sidewall modulation to $W_{g}=0.20 \mu \mathrm{m}$ while maintaining the longitudinal profile of the modulation. The transmission spectra for the 200 $\Lambda$-long asymmetric grating are compared with that of $W_{g}=0.1 \mu \mathrm{m}$ in Fig.3. Due to the rapidly oscillating sinusoidal carrier, only the envelopes of the signals are visible in the figure. The operating frequency for the nonlinear switching analysis was then chosen to be $214.03 \mathrm{THz}$. This frequency is closer to the band edge $(214.08 \mathrm{THz})$ than that of the previous case because the transmission spectrum is much steeper at the band edge, which allows the same level of extinction ratio for the nonlinear switching operation. The results for the switching behavior are shown in Fig.4 . Either of the two transmission plots show the Schmitt trigger operation, i.e. the switch turns on at a certain incident power, holding the on-state until the input passes through a lower threshold power. In this result the switching threshold has been reduced to $E_{t h}^{(o n)}=1.05 \times 10^{7} \mathrm{~V} / \mathrm{m}\left(P_{t h}^{(o n)}=77 \mathrm{~mW}\right)$ for the RTL (right to left, positive taper) configuration, and $E_{t h}^{(o n)}=1.6 \times 10^{7} \mathrm{~V} / \mathrm{m}\left(P_{t h}^{(o n)}=170 \mathrm{~mW}\right.$ ) for the LTR (left to right, negative taper) configuration. As expected, the stronger modulation allows the reduction of the switching threshold significantly. With the incident laser power of $77 \mathrm{~mW}$, the experimental observation of the switching operation in this device should be feasible.

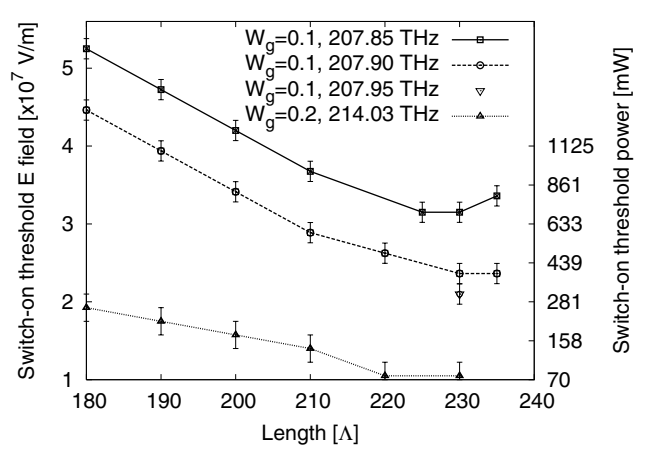

Fig.3 Switch-on threshold electric field and power versus the length of the waveguide grating for the RTL (right to left) configuration with $W_{g}=0.1 \mu \mathrm{m}$ and $0.2 \mu \mathrm{m}$. The error bars are the uncertainty due to the increment of the incident field variation.

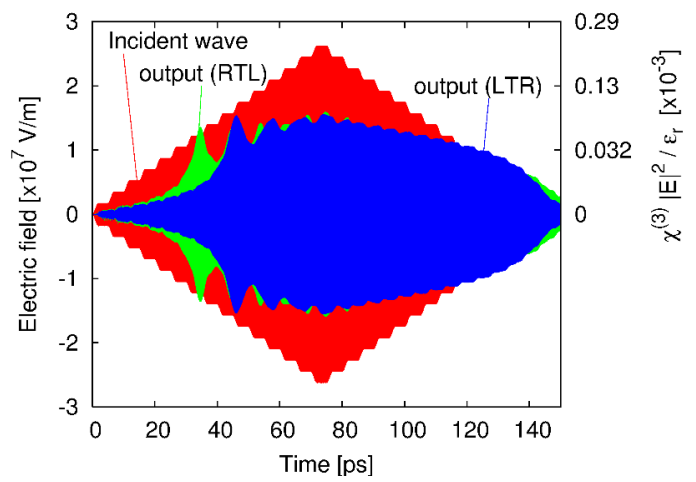

Fig.4 Switching for the LTR (left to right) and RTL configuration of a $220 \Lambda$-long waveguide with $W_{g}=$ $0.20 \mu \mathrm{m}$ at $214.03 \mathrm{THz}$ operating frequency $(\Delta f=$ $0.05 \mathrm{THz}$ ). Maximum incident field is $2.6 \times 10^{7} \mathrm{~V} / \mathrm{m}$ at 75 ps. Minimum switch-on threshold is $E_{t h}^{(o n)}=$ $1.05 \times 10^{7} \mathrm{~V} / \mathrm{m}\left(P_{t h}^{(o n)}=77 \mathrm{~mW}\right)$ for RTL.

\section{REFERENCES}

[1] W. Freude, A. Maitra, J. Wang, C. Koos, C. Poulton, M. Fujii, and J. Leuthold, "All-optical signal processing with nonlinear resonant devices", in 8th International Conference on Transparent Optical Networks, ICTON, Nottingham, UK, June 2006, We.D2.1.

[2] W.Chen and D.L.Mills, "Gap solitons and the nonlinear optical response of superlattices", Phys. Rev. Lett., vol. 58, no. 2, pp. 160-163, Jan. 1987.

[3] M.W.Feise, I.V.Shdrivov, and Y.S.Kivshar, "Bistable diode action in left-handed periodic structures", Phys. Rev. E, vol. 71, pp. $037602,2005$.

[4] X-H.Jia, Z-M.Wu, and G-Q.Xia, "Analysis of bistable steady characteristics and dynamic stability of linearly tapered nonlinear Bragg gratings", Opt. Exp., vol. 12, no. 13, pp. 2945-2953, June 2004.

[5] M.Fujii, C.Koos, C.Poulton, J.Leuthold, and W.Freude, "Nonlinear FDTD analysis and experimental verification of four-wave mixing in InGaAsP/InP racetrack micro-resonators", IEEE Photon. Technol. Lett., vol. 18, no. 2, pp. 361-363, Feb. 2006.

[6] C.M.de Sterke and J.E.Sipe, "Gap solitons", in Progress in Optics, vol.XXXIII, pp.203-260, North-Holland, Amsterdam, 1994.

[7] K.S.Yee, "Numerical solution of initial boundary value problems involving Maxwell's equation in isotropic media", IEEE Trans. Antennas Prop., vol. 14, no. 5, pp. 302-307, May 1966.

[8] M.Fujii, M.Tahara, I.Sakagami, W.Freude, and P.Russer, "High-order FDTD and auxiliary differential equation formulation of optical pulse propagation in 2D Kerr and Raman nonlinear dispersive media", IEEE J. Quantum Electron., vol. 40, no. 2, pp. 175-182, Feb. 2004. 